\title{
Examining Diagnostic Options and Classification Systems Available for Endometriosis
}

\author{
Authors: \\ *Debabrata Ghosh, Jayasree Sengupta \\ Department of Physiology, All India Institute of Medical Sciences, New Delhi, India \\ *Correspondence to debabrata.ghosh1@gmail.com \\ Disclosure: \\ The authors have declared no conflicts of interest. \\ Received: \\ 24.01.21 \\ Accepted: \\ 16.04.21 \\ Keywords: \\ Biomarkers, disease staging, early diagnosis, endometriosis, infertility, pelvic pain. \\ Citation: \\ EMJ Repro Health. 2021;7[1]:60-71.
}

\section{Abstract}

Introduction: Endometriosis is characterised by the presence of endometrium-like tissue outside the uterus, and is often associated with chronic pelvic pain, infertility, and compromised quality of life. Development of reliable methods of early diagnosis, staging, and classification of endometriosis would allow for restriction of disease progression by its early detection and strategising towards its early treatment and management.

Diagnostic options: Typically, diagnosis and staging of endometriosis include a history and physical examination followed by clinical, imaging, and laparoscopic findings. Surgical inspection of lesions at laparoscopy with histological confirmation remains the most reliable procedure towards the detection of endometriosis and its classification. Although there are many putative peripheral biomarkers having potential diagnostic values for endometriosis, further studies are necessary for their validation.

Classification systems: Based on anatomical, clinical, imaging, and several pathophysiological findings, various classifications and staging systems of endometriosis, e.g., revised American Society for Reproductive Medicine (rASRM), ENZIAN, Endometriosis Fertility Index (EFI) and Foci-Ovarian endometrioma-Adhesion-Tubal endometriosis-Inflammation (FOATI) scoring systems, have so far been postulated. However, there is no fool-proof diagnostic and classification approach available for the disease due to the general failure of current systems to reflect reproducible correlation with the major symptoms of endometriosis.

Conclusion: A 'toolbox approach', using all the available diagnostic and classification systems maximising the information available to healthcare providers and females, is a recent recommendation. Development of collaborative research networks for the harmonisation of patient information, biological sample collection, and its storage, and that of methodological and analytical tools in a wider patient base is necessary to discover reliable leads for future diagnostic options and a classification system for endometriosis. 


\section{INTRODUCTION}

Endometriosis is a complex gynaecological disorder characterised by the presence of endometrium-like tissue outside the uterus, primarily on pelvic organs, and affects approximately $10 \%$ of females of reproductive age. ${ }^{1}$ One out of two patients with endometriosis suffers from symptoms like painful periods (dysmenorrhea), non-menstrual chronic pelvic pain, pain due to intercourse (dyspareunia), and infertility ${ }^{2}$ There is little curative medical care. Surgical treatments often result in high rates of recurrence and loss of ovarian reserve, resulting in loss of fecundity'-3 The quality of life (QoL) in patients with this disease thus is significantly compromised, and it deteriorates even further due to the loss of productive time along with oft-present comorbidities, and resultant high healthcare spending ${ }^{2,4}$ Above all, the absence of robust diagnostic markers often results in delay of its early diagnosis and medical intervention. ${ }^{5}$ Endometriosis appears to be a disorder with a variegated pathophysiological basis and disease manifestation. Endometriosis may present as superficial peritoneal endometriosis, ovarian endometriosis (or endometrioma), and deep infiltrating endometriosis (or rectovaginal nodules) with discernible histological differences. ${ }^{6}$ Due to the inherent heterogeneity in phenotypes of endometriosis, the disease poses a serious challenge against attempts to improvise any simple operative approaches to the disease. Given the burden of individual stress, socio-economic strain, and clinician's anxiety linked to the disease, special attention for the development of non-invasive and minimally invasive but reliable diagnosis and classification of endometriosis appears a necessity. This would allow for the restriction of disease progression by its early detection and strategising towards its early treatment and management, and consequent avoidance of pain, stress, and invasive surgery. The aim of this article is to address the state of current knowledge regarding various diagnostic options as well as classification and staging systems available for endometriosis.

\section{DIAGNOSTIC OPTIONS}

The workup for diagnosis of endometriosis in a patient typically includes history noting

and physical examination followed by clinical and imaging investigations, and laparoscopic examinations. History and physical examinations yield non-specific, but occasionally useful, information. Familial tendency and history of pain and infertility, palpable tender nodular masses on pelvic examination, and elevated cancer antigen-125, along with infertility and/or chronic pelvic pain provide important but generally non-specific cues. Among imaging techniques, ultrasound and MRI bear some diagnostic value, though those are not sufficiently specific and sensitive to different types of endometriosis and non-endometriotic lesions. ${ }^{7-8}$ According to a Cochrane Database systematic review of imaging modalities for the non-invasive diagnosis of endometriosis, none of the imaging modalities were able to detect overall endometriosis with sufficient accuracy. ${ }^{9}$

Despite the fact that imaging may give useful indications, visual inspection of pelvic and extrapelvic lesions at laparoscopy with histological confirmation remains the most reliable procedure towards the detection of endometriosis and its classification. Symptomatic individuals having likelihood of endometriosis are recommended to undertake laparoscopic examination. One out of 4 women who undergo a laparoscopic procedure due to symptoms of suspected endometriosis does not show endometriosis. ${ }^{10}$ For a myriad of phenotypical issues, surgeons often face a diagnostic dilemma while inferring their observations. ${ }^{8}$ In addition, laparoscopic examination is often hindered by the presence of dense pelvic adhesions. ${ }^{8}$

Histological confirmation in terms of any of the two features, namely endometrial glands, endometrial stroma, and hemosiderin-laden macrophages is the prerequisite of definitive confirmation of disease after visualisation of lesions. Two out of three patients with lesions considered to be endometriosis on laparoscopic examination were not histologically confirmed. 8,10 Additionally, false-negative results are often reported in cases of atypical lesions with histologically confirmed endometriosis. ${ }^{10}$ Even with carefully conducted biopsy procedures by skilled surgeons and properly sampled specimens sent for pathologic examination, onequarter of biopsy samples do not turn out to be endometriosis. ${ }^{10,11}$ Nevertheless, laparoscopy is considered to be the standard modality for 
the diagnosis of endometriosis.7 Furthermore, laparoscopy can be applied for the treatments in which endometriomas may be cauterised or removed and adhesions can be lysed.? Laparoscopic surgery is, however, associated with an increased risk of intraoperative injury to bowel, bladder, ureter, and blood vessels.10,11 Table 1 summarises some of the cardinal advantages and disadvantages of the abovementioned different diagnostic methods., 710

Table 1: Different diagnostic tools and their advantages and limitations.

\begin{tabular}{|c|c|c|}
\hline Diagnostic tool & Advantage & Limitation \\
\hline TVUS & $\begin{array}{l}\text { - Minimally invasive. Accessible, inexpensive, } \\
\text { fast, and safe. } \\
\text { Allows real-time assessment of pain and } \\
\text { organ mobility. Particularly helpful for } \\
\text { ovarian and bladder endometriosis. } \\
\text { Useful in planning and ENZIAN and FOATI } \\
\text { scoring. }\end{array}$ & $\begin{array}{l}\text { - Highly operator-dependent. } \\
\text { - } \quad \text { Limited to the focal length of the probe. } \\
\text { ovarian lesions. } \\
\text { - Ovarian cysts, subserosal leiomyomas, and acute } \\
\text { retroflexion of the uterus, and also severe pelvic } \\
\text { adhesions and other distortions of the pelvic } \\
\text { anatomy may limit target visualisation. }\end{array}$ \\
\hline REUS & $\begin{array}{l}\text { - } \quad \text { Minimally invasive. } \\
\text { - } \quad \text { Useful for patients with suspected DIE. } \\
\text { - } \text { Provides a reliable method to evaluate } \\
\text { intestinal wall infiltration. } \\
\text { - Useful in ENZIAN and FOATI scoring. }\end{array}$ & $\begin{array}{l}\text { - } \quad \text { Highly operator-dependent. } \\
\text { - } \quad \text { Provides restricted view field. } \\
\text { - Not useful for assessing ovarian, peritoneal, or } \\
\text { anterior compartments. }\end{array}$ \\
\hline MRI & $\begin{array}{l}\text { - } \quad \text { Non-invasive. } \\
\text { Excellent soft-tissue contrast with } \\
\text { multiplanar capabilities and full panoramic } \\
\text { and simultaneous assessment of both } \\
\text { anterior and posterior compartments of } \\
\text { pelvic structures. } \\
\text { - Particularly useful in diagnosis of extensive } \\
\text { pelvic adhesions and deep infiltration. } \\
\text { - Useful in ENZIAN and FOATI scoring. }\end{array}$ & $\begin{array}{l}\text { - Highly specialised, expensive, lengthy procedures. } \\
\text { - } \quad \text { Limited use in patients with pacemakers or } \\
\text { cochlear implants, and with claustrophobia and/or } \\
\text { morbid obesity. } \\
\text { - } \quad \text { Real-time evaluation is rare. } \\
\text { - Bowel peristalsis may limit evaluation of intestinal } \\
\text { DIE. Stool and gas may limit DIE visualisation. }\end{array}$ \\
\hline
\end{tabular}

DIE: Deep infiltrating endometriosis; FOATI: foci-ovarian endometrioma- adhesion-tubal endometriosisinflammation; REUS: rectal endoscopic ultrasound; TVUS: transvaginal ultrasound.

Adapted from Espada et al. ${ }^{7}$ and Taylor et al. ${ }^{10}$

Given the insufficiency of available diagnostic tools, it is imperative that a biomarker-based approach may be devised to aid reliable diagnosis and classification of endometriosis. Typically, a biomarker is a characteristic that may be any substance, structure, or process in the body or its products and can be objectively measured and evaluated as an indicator of biological processes, normal or pathogenic, or pharmacologic responses to a therapeutic 
intervention. The measurable entity may be functional and physiological, biochemical at the cellular level, or a molecular interaction. ${ }^{12}$ The discovery of biomarkers with cues for diagnostic application potentially arises from a 'hypothesis driven' approach that screens a single molecule or a cohort of molecules involved in cardinal endometriosis-associated processes (e.g., neovascularisation, inflammation, cell survival, cell adhesion, cell proliferation and migration, and pain modulation) ${ }^{13}$ On the other hand, a 'screening -omics approach' employs a relatively 'hypothesis neutral' paradigm to investigate and analyse multiple parameters (e.g., mRNAs, non- coding RNAs [ncRNAs], proteins, peptides, lipids, and classes) that are considered to be associated with the development and pathogenesis of endometriosis. Of all possible peripherally obtainable biological samples, urine, blood, and endometrium appear to be the obvious choices. In the following section, an account of state-ofthe-art, clinically useful biomarkers as putative diagnostic options for endometriosis yielded from both 'hypothesis driven' and 'hypothesis neutral' approaches are presented. Table 2 provides a list of potential peripheral biomarkers of endometriosis. ${ }^{13-28}$

\section{Table 2: Potential peripheral biomarkers for endometriosis. ${ }^{13-28}$}

\begin{tabular}{|c|c|}
\hline Target material & Name of the molecule(s) \\
\hline Urine & $\begin{array}{ll}\text { - } & \text { Cytokeratin-19 (CK19) } \\
\text { - } & \text { Histone-4 } \\
\text { - } & \text { Soluble fms-like tyrosine kinase (sFlt)-1 } \\
\text { - } & \text { Vitamin D binding protein } \\
\end{array}$ \\
\hline Blood & $\begin{array}{ll}\text { - } & \text { a-1-B glycoprotein } \\
\text { - } & \text { Brain-derived neurotrophic factor } \\
\text { - } & \text { Chemokine ligands (CCLs)-2, }-5^{*} \\
\text { - } & \text { Chemokine ligand-8 } \\
\text { - } & \text { Glycodelin A } \\
\text { - } & \text { Haptoglobin } \\
\text { - } & \text { Hepatocyte growth factor } \\
\text { - } & \text { IL-1, -6, -8 } \\
\text { - } & \text { Matrix metalloproteinases-2, }-3,-9 \\
\text { - } & \text { Monocyte chemoattractant protein-1 } \\
\text { - } & \text { TNF-a } \\
\text { - } & \text { miRNA-28-5p, miR-29a-3p, 125b-5p }\end{array}$ \\
\hline Endometrium & $\begin{array}{l}\text { - } \text { Annexin-A2, -V } \\
\text { - } \\
\text { recepthroblastic leukaemia viral oncogene homologue } \\
\text { - } \\
\text { - } \\
\text { - } \\
\text { - } \\
\text { miRnatelet-derived growth factor receptor } \\
\text { miR- miR-100, miR-200a, miR-200b }\end{array}$ \\
\hline
\end{tabular}

*Also known at RANTES (Regulated on Activation, Normal T-cell Expressed and Secreted).

Markers may be specific to type and stage of the disease, and more correlated to specific symptom(s) of the disease (e.g., pain, infertility). None of these markers are fool-proof. A cohort of markers may present better specificity and sensitivity than any one of the biomarkers singularly. 


\section{Urinary Biomarkers}

In a Cochrane Database systematic review, an attempt was made to assess the diagnostic performances of non-neural enolase (enolase-1), vitamin $D$ binding protein, urinary peptide profiling, and cytokeratin-19. ${ }^{14}$ The review reportedly failed to identify any significant differences in individuals with endometriosis from a disease-free control group. ${ }^{14}$ In a comprehensive review analysing reported studies on the differential expression of urinary proteins as biomarkers of endometriosis, Gueye et al. drew a similar conclusion..$^{15}$ The results of a proteomic study indicated elevated histone 4 as a potential biomarker. ${ }^{16}$ Also, a combination of four urinary proteins, namely histone 4, ADPribosylation factor 3 , ribophorin 1 , and myosin heavy chain 10 reflects significant promise of diagnostic value. ${ }^{16}$ A secondary observation of the study that the high mobility group box 1 , cluster of differentiation 40 , and lymphotoxin $\beta$ receptor signalling pathways were activated in endometriosis appears interesting.

Since these signalling pathways are integral to the inflammation process, the notion that chronic inflammation might take part in the development of endometriosis is being corroborated by this observation..$^{29,30}$ Thus, the combined urinary proteins may have significant promise for yielding cues for diagnostic and therapeutic options, but this requires further robust validation.

\section{Circulatory Biomarkers}

The results obtained from a multi-centre study have indicated CA-125 $\geq 30$ unit/mL in peripheral circulation can act as a rule-in test for early diagnosis of endometriosis amongst women presenting with symptoms of pain and/ or subfertility. ${ }^{17}$ A multiplex profiling study of cytokines and angiogenic growth factors in plasma samples of patients with endometriosis and healthy controls revealed a potential panel of 14 cytokines (chemokine [C-C motif] ligand 2 [CCL2], CCL17, CCL21, CXCL5, CXCL11, CD14, carcinoembryonic antigen-related cell adhesion molecule 1 [CEACAM-1], erythroblastic leukaemia viral oncogene homologue 3 [ERBB3], IL-7, Lipocalin-2, neuronal cell adhesion molecule [NrCAM], receptor for advanced glycation end products [RAGE], TGF- $\beta$, and TNF- $\beta$ ) as a biomarker cohort with significance, specificity, and sensitivity to endometriosis disease samples. ${ }^{18}$ It is however noteworthy that nine cytokines (shown above) revealed only marginal $(p<0.05)$ differences in patients with endometriosis as compared to healthy controls and that a few (e.g., CCL21, IL-7, TGF- $\beta$ ) of those cytokines were seen to be differentially expressed in other inflammatory gynaecological disorders such as polycystic ovary syndrome, ovarian cysts, and pelvic adhesions. ${ }^{18}$ Further studies on larger sample sizes with confirmed disease phenotypes are necessary to reach a point of useful clinical diagnostic option. It is notable in this regard that a Cochrane Database systematic review of 70 studies evaluating 47 blood biomarkers (angiogenesis factors, growth factors, apoptosis markers, cell adhesion molecules, high-throughput markers, hormonal markers, immune system markers, inflammatory markers, oxidative stress markers, microRNAs, tumour markers, and other proteins) with meta-analyses performed for four markers (anti-endometrial antibodies, IL-6, CA-19.9, and CA-125) failed to differentiate people with endometriosis from disease-free controls. ${ }^{19}$

With pain being a common symptom of endometriosis, several studies were performed to examine whether neurotrophic molecules detected in blood can be used as diagnostic markers. Brain-derived neurotrophic factor (BDNF) in circulation as a putative marker was reportedly able to differentiate cases between Stage I and Stage II endometriosis. ${ }^{20}$ Although a higher serum level of mature BDNF was detected in those with self-reported pain with Stages III endometriosis prior to surgery, independent of menstrual cycle phase and irrespective of lesion type, the difference based on receiver operating characteristic curve analysis was not predictive for the disease..$^{21}$ In people with ovarian endometriosis and infertility with or without pain, BDNF levels in serum and peritoneal fluid were significantly higher in patients with pain but showed no association with the disease stages or menstrual cycle phases, however, correlated with BDNF mRNA and protein expression levels, and tyrosine receptor kinase B protein (receptor for BDNF and neurotrophin-3, -4 ligands) expressions in ectopic lesions in the presence of endometriosis pain. ${ }^{22}$

A large number of ncRNAs including microRNAs (miRNAs), long non-coding RNAs, and closed 
long non-coding circular RNAs are involved in tissue-specific regulation of gene expressions at the transcriptional, post-transcriptional, and translational levels. ${ }^{29}$ Thus, specific species of ncRNAs in peripheral biological samples, e.g., plasma, serum, saliva, and urine with high stability and pathophysiological relevance, bear potential biomarker value for various complex diseases, for example, endometriosis. ${ }^{23-26,32}$ However, no circulating ncRNA has as yet been identified that could on their own comprise a reproducible, non-invasive diagnostic test for endometriosis. The observed lack of concordance between the reported studies could include geographic and ethnic differences in the expression of ncRNA repertoire, differences in sample handling, ncRNA extraction, normalisation, assay platforms, methods of statistical analysis, and the absence of a harmonised approach to tissue collection, storage, and of specimen characterisation on the basis of disease severity, disease phenotype, menstrual history, and fertility. ${ }^{33-38}$

\section{Endometrial Biomarkers}

Eutopic endometrium from patients with endometriosis differs from that of those without endometriosis. ${ }^{13,27}$ It is commonly believed that endometrial biopsies collected using minimally invasive techniques with the aid of Pipelle or Karmen devices can be employed in the diagnosis of endometriosis. However, the dynamic nature of the cellular and molecular biology of endometrium, and additional complicating facets of the phenotypic and ethnic heterogeneity of endometriosis collectively pose challenges in the development of biomarker discovery for this disease. ${ }^{28,39}$ In fact, a close survey of the literature reveals multiple caveats that would require close attention in future studies aimed at developing eutopic endometrium-based diagnostic targets for endometriosis. In the following section, the authors highlighted a few important issues in this regard.

> The menstrual cycle phase of tissue collection is a strong variable since the ratios of various endocrine factors differentially influence the cellular expressions of biomolecules in endometrium under disease compared to normal conditions. $13,35,40$

> Potential biochemical differences in lesion subtypes of peritoneal, ovarian, and deep infiltrating endometriosis are reflected in studies comparing eutopic endometria to control tissues. ${ }^{13,41,42}$

> The fertility status of individual patients may influence endometrial expressions. ${ }^{37,43}$ Endometrial expressions may vary depending on the severity stages of disease in patients with positive fertility compared with infertile people with endometriosis. ${ }^{44,45}$

> The choice of endometriosis-free controls is an important issue since the presence of fibroids, adenomyosis, and/or pelvic organ prolapse may differentially affect the endometrial behaviour compared to that with no abnormality. ${ }^{46}$ Furthermore, the choice of endometriosis-free control with pain and without pain is likely to display distinctions in the molecular expressions. ${ }^{42,47}$ A high prevalence (approximately 45\%) of asymptomatic cases (no pain or other symptoms) of endometriosis in individuals may cast significant skew in the control data. ${ }^{48}$

> The heterogeneity of tissue components that include inflammatory cells, stromal cells, epithelial cells, endothelial cells in uterine wall components, surrounding peritoneal tissue in different biopsy specimens per se may affect tissue expressional repertoire of tissue..$^{34,38,49}$

Briefly, it appears that many peripheral biomarkers tentatively show promise in the diagnosis of endometriosis, but not a single biomarker or panel of biomarkers appears to be clinically fool-proof. It also appears that panels of markers rather than specific candidate markers may allow increased sensitivity and specificity for early diagnosis. Thus, after a decade of the reports based on systematic reviews of peripheral biomarkers of endometriosis by May et al., the present position remains similar: further research is warranted before any set of markers for endometriosis may be recommended for routine healthcare purposes. ${ }^{50}$

\section{CLASSIFICATION AND STAGING OF ENDOMETRIOSIS}

A reproducible classification system for a complex disease like endometriosis bears an advantage towards describing the pathological correlates of disease with acceptable levels of accuracy and precision, and also towards strategising effective medical and surgical interventions of the disease and disease-associated signs and symptoms. 
Additionally, a simple and user-friendly classification protocol would render great help for communication between clinicians and other stakeholders, including patients.

Endometriosis can be classified according to its primary nidus (peritoneal, ovarian, rectovaginal etc.). Sampson classified the endometrioma into follicular, corpus luteal, stromal, and endometrial types depending on the presence of haemorrhagic cysts and adjoining adhesions..$^{51}$ On the basis of anatomical location, clinical findings, and histology, endometriosis may present as Sampson's syndrome (infertility and/ or chronic dyspareunia with no deep pelvic local tenderness, induration or nodule formation, and histology showing superficial lesions of clear, red, black, or white lesions of endometriumlike glands and stroma), and Cullen's syndrome (tender palpable nodular or indurated lesion in the deep pelvis with histology of marked fibromuscular hyperplasia containing islands of endometrium-like glands).52 Endometriosis can also be classified as subtle, typical, cystic, deep, adenomyotic, and peritoneal pocket lesions estimated by their size.53 Ideally, a classification system should be able to identify disease morphology and severity with a high degree of accuracy and precision, and correlate the severity with the reported signs and symptoms of the disease (e.g., pain and subfertility).54 Accordingly, several attempts have been made to chronicle this evolving chronic disease in order to assess the stages and nature of lesions in association with pain scores and infertility.

In the following section, a summary of various classification systems available for endometriosis that include anatomical findings and disease staging based on imaging and laparoscopic investigations according to revised American Society for Reproductive Medicine (rASRM) and ENZIAN scores, and combinatorial approaches like Endometriosis Fertility Index (EFI) scoring and the FOATI systems is presented. Figure 1 provides the basic templates of the revised ASRM and ENZIAN protocols.

\section{Revised American Society for Reproductive Medicine Scoring System}

The American Fertility Society (AFS) introduced a scoring system for endometriosis in 1985 and a revised scoring procedure of the ASRM in
1996.55 According to rASRM, endometriosis is classified as superficial and deep lesions and staged as minimal (Stage I; Score: 1-5), mild (Stage II; Score: 6-15), moderate (Stage III; Score: 16-40), and severe (Stage IV; Score: $>40$ ). Some of the cardinal features of the rASRM scoring system are shown in Figure 1A. In the rASRM stages, weightage to endometriosis-associated visual landmarks at laparoscopy is attributed using arbitrarily designated scoring scales. This may lead to scoring of the disease to the same stage despite inherent differences in the nature of lesions, the latter having obvious bearing on strategising individual patient's treatment.56 The failure rate of such a protocol could reportedly be as high as 50\%, and it is around 20\% at best.57 Nevertheless, the rASRM protocol is widely practised for its ease to administer, report, and communicate, and for its apparent objective mode of presentation.

\section{ENZIAN Classification System}

This classification system was introduced to supplement the rASRM system, especially taking into account deep infiltrating endometriosis and its involvement with other organs.58 ENZIAN classification was named after Hotel Enzian on Lake Weissensee in the Austrian Alps, where the $7^{\text {th }}$ Conference of the Stiftung Endometriose Forschung (Foundation for Endometriosis Research), $25^{\text {th }}-27^{\text {th }}$ February 2011 developed this classification system. The original ENZIAN system was revised to reduce overlap with the rASRM system. In the revised ENZIAN classification system, the retroperitoneal structures are divided into three compartments: Compartment $A$ consists of the rectovaginal septum and vagina; Compartment B consists of the uterosacral ligament and pelvic walls; and Compartment $C$ consists of the sigmoid colon and rectum. The severity of the lesion is graded from its invasiveness (Grade 1: $<1 \mathrm{~cm}$; Grade 2: 1-3 cm; Grade 3: $>3 \mathrm{~cm}$ ). Deep endometriotic lesions in retroperitoneal distant locations (FA: adenomyosis; FB: involvement of the bladder; FU: intrinsic involvement of the ureter; $\mathrm{Fl}$ : bowel disease caudal to the rectosigmoid junction; and FO: other locations, such as abdominal wall endometriosis) are also indicated in the system. A succinct coverage of the ENZIAN classification system is available in the 2020 recommendation of the Working group of the European Society 
for Gynaecological Endoscopy (ESGE), the Endometriosis Society (WES).59 Some of the European Society of Human Reproduction cardinal features of ENZIAN system are shown and Embryology (ESHRE), and the World in Figure 1B.

\begin{tabular}{|c|c|c|c|}
\hline Endometriosis & $<1 \mathrm{~cm}$ & $1-3 \mathrm{~cm}$ & $>3 \mathrm{~cm}$ \\
\hline Peritoneum superficial & 1 & 2 & 4 \\
\hline Peritoneum deep & 2 & 4 & 6 \\
\hline Right ovary superficial & 1 & 2 & 4 \\
\hline Right ovary deep & 4 & 16 & 20 \\
\hline Left ovary superficial & 1 & 2 & 4 \\
\hline Left ovary deep & 4 & 16 & 20 \\
\hline \multicolumn{4}{|c|}{ Posterior cul-de-sac obliteration } \\
\hline \multicolumn{2}{|l|}{ Partial } & \multicolumn{2}{|l|}{4} \\
\hline \multicolumn{2}{|l|}{ Complete* } & \multicolumn{2}{|l|}{40} \\
\hline Adhesions $^{\dagger}$ & $\begin{array}{l}<1 / 3 \\
\text { enclosure }\end{array}$ & $\begin{array}{l}1 / 3-2 / 3 \\
\text { enclosure }\end{array}$ & $\begin{array}{l}>2 / 3 \\
\text { enclosure }\end{array}$ \\
\hline Right ovary filmy & 1 & 2 & 4 \\
\hline Right ovary dense & 4 & 8 & 16 \\
\hline Left ovary filmy & 1 & 2 & 4 \\
\hline Left ovary dense & 4 & 8 & 16 \\
\hline Right tube filmy & 1 & 2 & 4 \\
\hline Right tube dense & 4 & 8 & 16 \\
\hline Left tube filmy & 1 & 2 & 4 \\
\hline Left tube dense & 4 & 8 & 16 \\
\hline
\end{tabular}


(B)

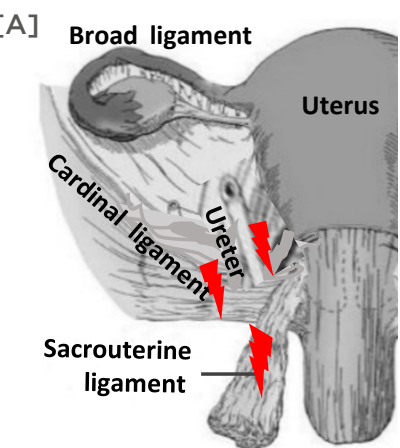

[B]

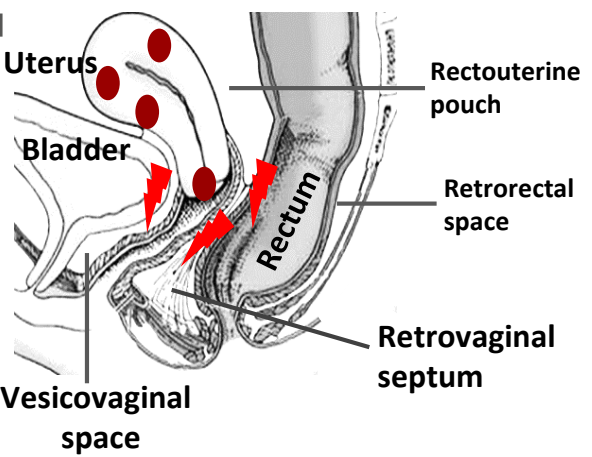

$[\mathrm{C}]$

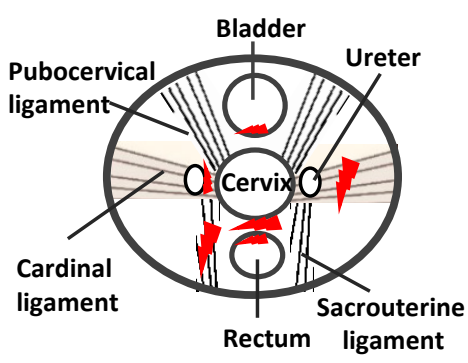

(C)

\begin{tabular}{|l|l|l|l|}
\hline Compartment & A & B & C \\
\hline $\begin{array}{l}\text { Anatomical } \\
\text { localisation }\end{array}$ & $\begin{array}{l}\text { Rectovaginal space } \\
\text { vagina }\end{array}$ & $\begin{array}{l}\text { Sacrouterine and cardinal } \\
\text { ligaments } \\
\text { External ureter } \\
\text { compression }\end{array}$ & $\begin{array}{l}\text { Sigmoid colon } \\
\text { Rectum }\end{array}$ \\
\hline & &
\end{tabular}

\section{Figure 1:}

A) Revised American Society for Reproduction Medicine (rASRM) scoring system for staging of endometriosis. ${ }^{55}$ Determination of the stages of endometriosis based on examination of the pelvis at laparoscopy in a clockwise or counter-clockwise manner to note the number, size, and location of endometrial implants, plaques, endometriomas, and adhesions. The surface of the uterus is considered as peritoneum. Adhesions and lesions in the peritoneum, ovary, fallopian tubes, uterus, and cul-de-sac are scored as shown. Superficial peritoneal implants are shown as red, red-pink, flame-like, vesicular blobs. Clear vesicles, white opacifications, or haemosiderin deposits seen as black, blue deposits or yellow-brown deposits are also detected and scored. Adhesions are seen as filmy or dense, covering the ovary and tubes extending to the cul-de-sac, as shown in the different stages of endometriosis.

B) ENZIAN scoring system for deep infiltrating endometriosis. ${ }^{59}$ The ENZIAN scoring provides a scoring of deep infiltrating endometriosis in retroperitoneal structures based on laparoscopic identification and the use of imaging (transvaginal ultrasonography, rectal endoscopic ultrasonography, MRI) techniques. The ENZIAN scores include lesions in the cul-de-sac, vagina, cervico-uterine ligaments, bladder, ureter, bowel, and uterus. The major anatomical sites of endometriotic lesions are sacrouterine ligament, cardinal ligament, and ureter [A]. Adenomyosis lesion sites in the uterus with the presence of heterotopic endometrial glands and stroma in the myometrium and reactive fibrosis of the surrounding smooth muscle cells of the myometrium, which often co-exists with endometriosis. Endometriotic lesion sites present in retrovaginal septum, bladder, vesicovaginal space, and retrorectal space [B] are shown. A schematic presentation of lesion sites detected within the pelvic compartment as shown in [A] and [B], excluding adenomyotic lesions, is presented in [C].

C) ENZIAN scoring system showing the levels (1-3) of deep endometriosis lesions that may be present in compartments like the rectovaginal space and vagina (A1-A3), sacrouterine, and cardinal ligaments to cause compression of external ureter wall (B1-B3) and the rectum (C1-C3). Other major lesion sites include adenomyosis (FA), lesions on the bladder (FB) and the ureter (FU). Endometriotic lesions may also be detected in extragenital sites such as the intestine, lung, and diaphragm, and in inguinal regions.

*Complete closure of the cul-de-sac by dense adhesions extending from the ovary and tube is scored as 40 . The aggregation of points as shown indicates the endometriosis disease stages as minimal (I), mild (II), moderate (III), or severe (IV).

+ Complete closure of the fimbriated end of the tube by adhesions is scored as 16.

Adapted from American Society for Reproductive Medicine 55 and Working Group of ESGE, ESHRE, and WES, Keckstein $\mathrm{J}$ et al. ${ }^{59}$ 
ENZIAN classification can be determined by imaging modalities and used for surgical planning, and it provides detailed descriptions of compartment-wise severity of lesion in the retroperitoneal structures, reportedly associated and correlated with the presence and severity of different symptoms (e.g., pain). ${ }^{60,61}$ The ENZIAN classification system has as yet received only moderate reception, primarily due to its not being a user-friendly protocol and for not having an easy communication gait; the system is both complex and employs complicated terminologies. Also, there are currently no sufficient evidencebased reports regarding the usefulness of the ENZIAN classification system in determining preoperative prediction regarding surgical decision.

\section{Combinatorial Approaches: \\ Endometriosis Fertility Index and Foci- Ovarian Endometrioma- Adhesion- Tubal Endometriosis-Inflammation- Adenomyosis- Recto-Vaginal Space System}

Infertility is one major issue affecting the QoL of a large percentage of patients with endometriosis. The EFI provides a classification system on the basis of scores obtained from the assessment of surgical factors and historical factors, and projects to predict the clinical outcome of pregnancy in patients who are infertile ${ }^{2,62} \mathrm{EFI}$ is considered a valid clinical tool to predict fertility outcome for people following surgical staging of endometriosis and may be used for developing suitable treatment plans for infertile women with endometriosis. 2,62,63

The Foci-Ovarian endometrioma- AdhesionTubal endometriosis-Inflammationadenomyosis- Recto-Vaginal Space (FOATIaRVS) system of classification takes into consideration the histology of ectopic lesions and functional repercussions for tubal and ovarian functions along with the nature of inflammation. Collectively, it may help to identify the nature of medical and surgical treatments to be undertaken in patients who are infertile and have endometrioma; and the chances of malignant proliferation. 64,65

To date, the authors have no template to classify 'atypical endometriosis', which is an intermediate precursor lesion linking typical endometriosis and clear cell/endometrioid tumours observed in 1-3 patients out of 100 endometriosis patients with endometrioma. ${ }^{66}$ DIE, affecting $1-2 \%$ of individuals of reproductive age also bears the risk of developing malignancy. ${ }^{67}$ Development of a classification system for assessment of endometriosis as a pre-malignant field defect which can be used for pre-emptive monitoring and management of the disease in a high-risk vulnerable population is seriously warranted. ${ }^{68}$

\section{PRACTICAL PERSPECTIVE AND RECOMMENDATIONS}

There is no fool-proof diagnostic option and classification or staging system for endometriosis disease. The core problem exists in the general failure of current systems to reflect a reproducible correlation with symptoms: infertility and pain, especially with the differential nature and severity of pain associated with endometriotic lesions in different compartments. Central and peripheral neural sensitisation and inflammation is causally associated with the pain caused from endometriosis, independent of anatomical distortion.

Conventional approaches to classifying endometriosis-associated pain based on disease, duration, and anatomy are grossly inadequate. Additionally, available diagnostic measures and classification systems fail to predict responses to medical and surgical interventions, disease recurrence, risks for associated disorders including malignancy, QoL measures, and other endpoints important to patients and healthcare providers for guiding appropriate therapeutic options and prognosis. In the given situation of a dearth of reliable diagnostic and classification systems, the WES recommends that clinicians adopt a 'toolbox approach' using all available diagnostic and classification systems, as appropriate, to maximise the information available to healthcare providers and patients.

It appears that the development of collaborative research study networks to harmonise the protocols for patient information, biological sample collection and their storage, and methodological and analytical tools, as well as applying those protocols to a wider patient base of diverse ethnicity and population is needed. This effort would pave the way to discovering reliable leads for future diagnostic options and a 


\section{References}

1. Bulun $\mathrm{S}$ et al. Endometriosis. Endocr Rev. 2019;40(4):1048-79.

2. Ghosh D et al. Pathophysiological basis of endometriosis-linked stress associated with pain and infertility: a conceptual review. Reprod Med. 2020;1(1):32-61.

3. Coccia EM et al. Bilateral endometrioma excision: surgeryrelated damage to ovarian reserve. Reprod Sci. 2019;26(4):543-50.

4. Soliman AM et al. Real-world evaluation of direct and indirect economic burden among endometriosis patients in the United States. Adv Ther. 2018;35(3):408-23.

5. Parasar $P$ et al. Endometriosis: epidemiology, diagnosis and clinical management. Curr Obstet Gynecol Rep. 2017:6(1):34-41.

6. Nisolle $\mathrm{M}$ et al. Peritoneal endometriosis, ovarian endometriosis, and adenomyotic nodules of the rectovaginal septum are three different entities. Fertil Steril. 1997;68(4):585-96.

7. Espada $M$ et al. Imaging techniques in endometriosis. J Endometriosis Pelvic Pain Disorders. 2018;10(3):136-50.

8. Berker B, Seval M. Problems with the diagnosis of endometriosis. Women's Health (Lond). 2015;11(5):597-601.

9. Nisenblat $\mathrm{V}$ et al. Imaging modalities for the non-invasive diagnosis of endometriosis. Cochrane Database Syst Rev. 2016;2(2):CD009591.

10. Taylor HS et al. An evidence-based approach to assessing surgical versus clinical diagnosis of symptomatic endometriosis. Int J Gynecol Obstet 2018;142(2):131-42.

11. Agarwal SK et al. Clinical diagnosis of endometriosis: a call to action. Am J Obstet Gynecol. 2019;220(4):354-64.

12. Strimbu K, Tavel JA. What are biomarkers? Curr Opin HIV AIDS. 2010;5(6):463-6

13. Sengupta J et al. "Molecular Biology of Endometriosis", Schatten $\mathrm{H}$ (ed.), Human Reproduction: Updates and New Horizons (2017), New York: John Wiley \& Sons, pp.71-141.

14. Liu $\mathrm{E}$ et al. Urinary biomarkers for the non-invasive diagnosis of endometriosis. Cochrane Database Syst Rev. 2015;2015(12):CD012019.

15. Gueye N-A et al. "Biomarkers for endometriosis in saliva, urine and peritoneal fluid", D'Hooghe T (ed.), Biomarkers for Endometriosis - State of Art (2017), New Delhi: Springer
India, pp.141-63.

16. Chen $X$ et al. Elevated urine histone 4 levels in women with ovarian endometriosis revealed by discovery and parallel reaction monitoring proteomics. J Proteomics. 2019;204(7):103398.

17. Hirsch $M$ et al. Diagnostic accuracy of cancer antigen 125 (CA125) for endometriosis in symptomatic women: a multi-center study. Eur J Obstet Gynecol Reprod Biol. 2017;210(3):102-7.

18. Weisheng B et al. Discovering endometriosis biomarkers with multiplex cytokine arrays. Clin Proteom. 2019;16(7):28.

19. Nisenblat $\mathrm{V}$ et al. Blood biomarkers for the non-invasive diagnosis of endometriosis. Cochrane Database Syst Rev. 2016;2016(5):CD012179.

20. Wessels JM et al. Assessing brainderived neurotrophic factor as a novel clinical marker of endometriosis. Fertil Steril. 2016;105(1):119-28.

21. Perricos A et al. Increased serum levels of mBDNF in women with minimal and mild endometriosis have no predictive power for the disease. Exp Biol Med (Maywood) 2018;243(1):50-6.

22. Ding $\mathrm{S}$ et al. Role of brain-derived neurotrophic factor in endometriosis pain. Reprod Sci. 2018;25(7):1045-57.

23. Zhang $M$ et al. Expression profile analysis of circular RNAs in ovarian endometriosis by microarray and bioinformatics. Med Sci Monit. 2018;24(12):9240-50.

24. Khalaj $\mathrm{K}$ et al. Extracellular vesicles from endometriosis patients are characterized by a unique miRNAIncRNA signature. JCl Insight. 2019;4(18):e128846.

25. Nisenblat $\vee$ et al. Plasma miRNAs display limited potential as diagnostic tools for endometriosis. J Clin Endocrinol Metab. 2019;104(6):19992022.

26. Vanhie A et al. Plasma miRNAs as biomarkers for endometriosis. Hum Reprod. 2019;34(9):1650-60.

27. May KE et al. Endometrial alterations in endometriosis: a systematic review of putative biomarkers. Hum Reprod Update. 2011;17(5):637-53.

28. Ahn $\mathrm{SH}$ et al. Biomarkers in endometriosis: challenges and opportunities. Fertil Steril. 2017:107(3):523-32.

29. McDaniel DK et al. Emerging roles for non-canonical NF-kB signaling in the modulation of inflammatory bowel disease pathobiology. Inflamm Bowel Dis. 2016;22(9):2265-79.

30. Lin $\mathrm{YH}$ et al. Chronic niche inflammation in endometriosisassociated infertility: Current understanding and future therapeutic strategies. Int J Mol Sci. 2018;19(8):2385.

31. Patil VS et al. Gene regulation by noncoding RNAs. Crit Rev Biochem Mol Biol. 2014;49(1):16-32.

32. Bhome R et al. Exosomal microRNAs (exomiRs): Small molecules with a big role in cancer. Cancer Lett. 2018;420(4):228-35

33. Becker CM et al. World Endometriosis Research Foundation Endometriosis Phenome and Biobanking Harmonisation Project: I. Surgical phenotype data collection in endometriosis research. Fertil Steril. 2014;102(5):1213-22.

34. Saare $M$ et al. Challenges in endometriosis miRNA studies - from tissue heterogeneity to disease specific miRNAs. Biochim Biophys Acta Mol Basis Dis. 2017;1863(9):2282-92.

35. Faraldi $\mathrm{M}$ et al. Free circulating miRNAs measurement in clinical settings: the still unsolved issue of the normalization. Adv Clin Chem. 2018;87(8):113-39.

36. Gevaert $A B$ et al. MicroRNA profiling in plasma samples using aPCR arrays: recommendations for correct analysis and interpretation. PLoS One. 2018;13(2):e0193173.

37. Anupa G et al. An assessment of the multifactorial profile of steroidmetabolizing enzymes and steroid receptors in the eutopic endometrium during moderate to severe ovarian endometriosis. Reprod Biol Endocrinol. 2019;17(12):111.

38. Anupa $\mathrm{G}$ et al. Endometrial stromal cell inflammatory phenotype during severe ovarian endometriosis as a cause of endometriosis associated infertility. Reprod Biomed Online. 2020;41(4):623-39

39. Bougie $\mathrm{O}$ et al. Influence of race/ ethnicity on prevalence and presentation of endometriosis: a systematic review and meta-analysis. BJOG. 2019;126(9):1104-15.

40. Huhtinen $\mathrm{K}$ et al. Endometrial and endometriotic concentrations of estrone and estradiol are determined by local metabolism rather than circulating levels. J Clin Endocrinol 
Metab. 2012;97(11):4228-35.

41. Braza-Boïls A et al. MicroRNA expression profile in endometriosis: its relation to angiogenesis and fibrinolytic factors. Hum Reprod. 2014;29(5):978-88.

42. Haikalis ME et al. MicroRNA expression pattern differs depending on endometriosis lesion type. Biol Reprod. 2018;98(5):623-33.

43. Leach RE et al. High throughput, cell type-specific analysis of key proteins in human endometrial biopsies of women from fertile and infertile couples. Hum Reprod. 2012;27(3):81428.

44. Khan MA et al. Genome-wide expressions in autologous eutopic and ectopic endometrium of fertile women with endometriosis. Reprod Biol Endocrinol. 2012 Sep 24;10:84.

45. Aghajanova L, Giudice LC. Molecular evidence for differences in endometrium in severe versus mild endometriosis. Reprod Sci. 2011;18(3):229-51.

46. Tamaresis JS et al. Molecular classification of endometriosis and disease stage using high-dimensional genomic data. Endocrinology. 2014:155(12):4986-99.

47. Sasamoto $\mathrm{N}$ et al. Evaluation of CA125 in relation to pain symptoms among adolescents and young adult women with and without surgicallyconfirmed endometriosis. PLoS One. 2020:15(8):e0238043.

48. Gylfason JT et al. Pelvic endometriosis diagnosed in an entire nation over 20 years. Am J Epidemiol. 2010;172(3):237-43.

49. Saare $M$ et al. DNA methylation alterations - potential cause of endometriosis pathogenesis or a reflection of tissue heterogeneity? Biol Reprod. 2018:99(2):273-82.

50. May KE et al. Peripheral biomarkers of endometriosis: a systematic review. Hum Reprod Update. 2010;16(6):65174.
51. Sampson JA. Perforating hemorrhagic (chocolate) cysts of the ovary. Their importance and especially their relation to pelvic adenomas of the endometrial type ('adenomyoma' of the uterus, rectovaginal septum, sigmoid, etc.). Arch Surg. 1921;3:245-323.

52. Garry R. The endometriosis syndromes: a clinical classification in the presence of aetiological confusion and therapeutic anarchy. Hum Reprod. 2004:19(4):760-68.

53. Koninckx PR et al. An endometriosis classification, designed to be validated. Gynecol Surg. 2011;8(10):16.

54. Abrao MS, Miller CE. An endometriosis classification, designed to be validated. NewsScope 2012;25(4):6.

55. American Society for Reproductive Medicine. Revised American Society for Reproductive Medicine classification of endometriosis: 1996. Fertil Steril. 1997;67(5):817-21.

56. Andres MP et al. Endometriosis classification according to pain symptoms: can the ASRM classification be improved? Best Pract Res Clin Obstet Gynaecol. 2018 Aug:51:111-18.

57. Fernando $S$ et al. Reliability of visual diagnosis of endometriosis. J Minim Invasive Gynecol. 2013;20(6):783-9.

58. Haas D et al. The rASRM score and the Enzian classification for endometriosis: their strengths and weaknesses. Acta Obstet Gynecol Scand. 2013;92(1):3-7.

59. Working Group of ESGE, ESHRE, and WES, Keckstein $\mathrm{J}$ et al. Recommendations for the surgical treatment of endometriosis. Part 2: deep endometriosis. Hum Reprod Open. 2020 Feb;2020(1):hoaaOO2.

60. Di Paola $\vee$ et al. Detection and localization of deep endometriosis by means of MRI and correlation with the ENZIAN score. Eur J Radiol. 2015:84(4):568-74.
61. Montanari E et al. Association between disease extent and pain symptoms in patients with deep infiltrating endometriosis. Reprod Biomed Online. 2019;39(5):845-51.

62. Adamson GD, Pasta DJ. Endometriosis fertility index: the new, validated endometriosis staging system. Fertil Steril. 2010;94(5):160915.

63. Johnson NP et al. World Endometriosis Society consensus on the classification of endometriosis. Hum Reprod. 2017;32(2):315-24.

64. Tran DK, Belaisch J. Is it time to change the ASRM classification for endometriosis lesions? Proposal for a functional FOATIaRVS classification. Gynecol Surg. 2012;9(3):369-73.

65. Bouquet de Joliniere et al. Is it necessary to purpose an add-on to the American classification of endometriosis? This disease can be compared to a malignant proliferation while remaining benign in most cases. EndoGram ${ }^{\circledast}$ is a new profile witness of its evolutionary potential. Front Surg. 2019;6(6):27.

66. Vercellini P et al. Perimenopausal management of ovarian endometriosis and associated cancer risk: When is medical or surgical treatment indicated? Best Pract Res Clin Obstet Gynaecol. 2018;51(8):15168.

67. Vilches Jimenez JC et al. Diagnostic challenges: low-grade adenosarcoma on deep endometriosis. BMC Women's Health. 2019;19(10):124.

68. Ghosh D et al. How benign is endometriosis: multi-scale interrogation of documented evidence. Cur Op Gyn Obs. 2019;2(1):318-45 